\title{
Mathematical Modeling of the Adaptive Immune Responses in the Early Stage of the HBV Infection
}

\author{
Karam Allali (D), ${ }^{1}$ Adil Meskaf, ${ }^{1}$ and Abdessamad Tridane ${ }^{(D)}{ }^{2}$ \\ ${ }^{1}$ Department of Mathematics, Faculty of Sciences and Technologies, Hassan II Mohammedia University, Mohammedia, Morocco \\ ${ }^{2}$ Department of Mathematical Sciences, United Arab Emirates University, P.O. Box 15551, Al Ain, UAE \\ Correspondence should be addressed to Abdessamad Tridane; a-tridane@uaeu.ac.ae
}

Received 29 August 2017; Revised 18 November 2017; Accepted 18 December 2017; Published 1 February 2018

Academic Editor: Ahmed M. Elaiw

Copyright (c) 2018 Karam Allali et al. This is an open access article distributed under the Creative Commons Attribution License, which permits unrestricted use, distribution, and reproduction in any medium, provided the original work is properly cited.

\begin{abstract}
The aim of this paper is to study the early stage of HBV infection and impact delay in the infection process on the adaptive immune response, which includes cytotoxic T-lymphocytes and antibodies. In this stage, the growth of the healthy hepatocyte cells is logistic while the growth of the infected ones is linear. To investigate the role of the treatment at this stage, we also consider two types of treatment: interferon- $\alpha$ (IFN) and nucleoside analogues (NAs). To find the best strategy to use this treatment, an optimal control approach is developed to find the possibility of having a functional cure to HBV.
\end{abstract}

\section{Introduction}

It is very well known that the adaptive immune response has a significant impact on the progress of the early stage of $\mathrm{HBV}$ [1]. This response can either lead to complete cure from the infection, and it is characterised by the production of neutralizing antibodies against $\mathrm{HBV}$ surface antigen (HBsAg) and adequate cytotoxic lymphocyte T-cell (CTL) responses [2-4], or it could result in chronic infection that leads to liver cancer (HHC), cirrhosis, or liver failure.

During the incubation period of $\mathrm{HBV}$, which is 30 to 180 days, the dynamics of the adaptive immune response are not fully understood, since the majority of the cases are clinically known after the infection is established and the patient is in the acute stage [5]. Understanding the dynamics of the two main arms of the adaptive immune response, CTL cells and Bcells $[6,7]$, will help grasp how the virus escapes the adaptive immunity and improve the ability of the immune system to control the virus in early infection.

Moreover, it is known that the actual therapy, which includes the standard interferon- $\alpha$ (INF) and the nucleoside analogues (NAs), is also initiated during the acute stage of HBV infection. INF helps eliminate the infected cells by reducing cccDNA [8], while NAs' function is to elongate DNA which leads to the inhibition of HBV replication
[8]. As monotherapy, the NAs come in different types of drugs (Entecavir, Adefovir, and Lamivudine), which are also known for enhancing the functions of natural killers [8]. The question is, what if we could initiate therapy even earlier? Is it possible to eradicate the virus within this period with the therapy? In fact, recent studies $[9,10]$ showed that early Lamivudine treatment could lead to better outcome in acuteon-chronic stages and with less liver damage. Therefore, our goal is to understand the dynamics of the adaptive immune response, via the CTL cells and B-cells, in the early stage of $\mathrm{HBV}$, and investigate the impact of early HBV treatment therapy on disease progress via a mathematical model of virus-immune response.

Mathematical modeling of the immune response to HBV is a subject that has been heavily investigated over the years by many authors [11-18], just to name a few. To our knowledge, there is no study that investigates the adaptive immune response in the early stage of the infection and effect of the early treatment on the progress of the disease. In this work, we are aiming to investigate this issue by considering an augmented model of our recent works [18, 19], and we consider the logistic growth only for the healthy hepatocyte cells and the infected hepatocyte cells [11]. This assumption is made to reflect the nature of the growth of these two types of cells in the early stage of the infection. We also did not 
consider the latently infected cells, which are established at an acute stage [11], and we did not consider noncytolytic carrying processes since no data support such assumption in this stage. Moreover, we have also considered a more generalized incident function [16] and the delay in this incident function to reflect the time between the infection and the cells becoming productively infected (infected and producing new viruses). The optimal control of the HBV therapy aims to find the optimal strategy of the drugs that allow blocking the virus production and infection. Several papers studied the optimal control of the HBV therapy [16, 20-22]. In our case, the therapy will have an antiviral effect, and we ignore its immunomodulatory effect since we do not know what impact the use of the therapy could have on the immune system in the early stage of HBV infection.

The paper is organized as follows. In Section 2, we introduce our model, and we investigate the basic properties of the model without therapy, which includes positivity and boundedness of solutions. In Section 3, we focus on the stability analysis of the different types of steady states. Next, we will investigate optimal control of the treatment therapy, and we will numerically solve the optimality conditions. Finally, we will give a discussion and a conclusion to our work.

\section{Introducing the Model}

We defined the dynamics of the early stage of the HBV by the following system:

$$
\begin{aligned}
\frac{d x}{d t}= & r x(t)\left(1-\frac{T(t)}{T_{m}}\right)-\beta\left(1-u_{1}(t)\right) \frac{v(t) x(t)}{T(t)} \\
\frac{d y}{d t}= & \beta e^{-k \tau}\left(1-u_{1}(t)\right) \frac{v(t-\tau) x(t-\tau)}{T(t-\tau)}-a y(t) \\
& -p y(t) z(t) \\
\frac{d v}{d t}= & \left(1-u_{2}(t)\right) a N y(t)-\delta v(t)-q v(t) w(t), \\
\frac{d w}{d t}= & g v(t) w(t)-h w(t) \\
\frac{d z}{d t}= & c y(t) z(t)-b z(t)
\end{aligned}
$$

with

$$
T(t)=x(t)+y(t),
$$

where $x(t), y(t), v(t), w(t)$, and $z(t)$ denote the concentrations of uninfected cells, infected cells, viruses, antibodies, and cytotoxic T-lymphocytes (CTLs), respectively. The uninfected hepatocytes grow at a rate that depends on the liver size, $T_{m}$, at a maximum per capita proliferation rate $r$. The healthy hepatocytes become infected by the virus at rate $\beta(v x / T)$, where $\beta$ is a constant. Infected cells $y$ die at rate $a$ and are killed by the CTLs response at rate $p$. The infected non-virus-producing cells have a death rate $k$; these cells start producing viruses after delay time $\tau$, hence $e^{-k \tau}$ is the probability of survival between time $t-\tau$ and $t$. The free virus particles are produced at rate $a N$, where $N$ is the number of free virions produced by the infected cells during their lifespan, and decay at rate $\delta$. Parameter $c$ represents the rate of expansion of CTL cell $z$ and $b$ is its decay rate in the absence of antigenic stimulation. The antibodies are developed in response to free virus at rate $g$ and decay at rate $h$. Finally, $u_{1}$ represents the efficiency of IFN in reducing the new infected cells; the infection rate in the presence of the drug is $\left(1-u_{1}\right) \beta$, while $u_{2}$ is the efficiency of NAs in blocking the reverse transcription, such that the virions production rate under this drug is $\left(1-u_{2}\right) a N$.

First, we analyse the model without drug therapy (i.e., $u_{1}=u_{2}=0$ ). More precisely, we consider the following model:

$$
\begin{aligned}
& \frac{d x}{d t}=r x(t)\left(1-\frac{T(t)}{T_{m}}\right)-\beta \frac{v(t) x(t)}{T(t)}, \\
& \frac{d y}{d t}=\beta e^{-k \tau} \frac{v(t-\tau) x(t-\tau)}{T(t-\tau)}-a y(t)-p y(t) z(t), \\
& \frac{d v}{d t}=a N y(t)-\delta v(t)-q v(t) w(t), \\
& \frac{d w}{d t}=g v(t) w(t)-h w(t), \\
& \frac{d z}{d t}=c y(t) z(t)-b z(t) .
\end{aligned}
$$

Let $X=C\left([-\tau, 0] ; \mathbb{R}^{5}\right)$ be the Banach space of continuous mapping from $[-\tau, 0]$ to $\mathbb{R}^{5}$ with respect to the norm

$$
\|\varphi\|=\sup _{-\tau \leq t \leq 0} \varphi(t) .
$$

We assume that the initial functions of the system of delayed differential equations (3) verify

$$
(x(t), y(t), v(t), z(t), w(t)) \in X .
$$

Following the standard approach, we assume that

$$
\begin{aligned}
& x(t)>0, \\
& y(t) \geq 0, \\
& v(t) \geq 0, \\
& z(t) \geq 0, \\
& w(t) \geq 0, \\
& T_{m} \geq T(t)=x(t)+y(t)>0, \quad \text { for } t \in[-\tau, 0],
\end{aligned}
$$

Under these initial conditions, the solutions of (3) satisfy the following theorem.

Theorem 1. System (3) has a unique solution; in addition, this solution is nonnegative and bounded for all $t \geq 0$. 
Proof. Notice that system (3) is locally Lipschitzian at $t=$ 0 , which implies that the solution of system (3), subject to (7), exists and is unique on $[0, b)$, where $b$ is the maximal existence time for the solution of system (3). Notice that if $x(0)=0$, then $x(t) \equiv 0$ for all $t>0$. Hence, we can assume that $x(0)>0$. Notice also that if $y(0)=0$, then, from (6), we have $y^{\prime}(0)=\beta((v(-\tau) x(-\tau)) / T(-\tau)) \geq 0 t$, which implies that, for small $t>0$, we have $y(t)>0$. Similarly, if $v(0)=0$, then $v^{\prime}(0)=a N y(0)>0$, which implies that, for small $t>0$, we have $v(t)>0$. Moreover, if $w(0)=0$, $z(0)=0$, then $w(t) \equiv 0, z(t) \equiv 0$ for all $t>0$. Hence, we assume below that $w(0)>0, z(0)>0$.

Assume first that there is $b>t_{1}>0$ such that $x\left(t_{1}\right)=$ 0 and $x(t)>0, y(t)>0, v(t)>0$, for $t \in\left[0, t_{1}\right]$. Observe that

$$
\frac{d x(t)}{d t}=r x(t)\left(1-\frac{T(t)}{T_{m}}\right)-\frac{\beta v(t) x(t)}{T(t)}
$$

it is easy to show that $0<T(t)<T_{m}$ for $t \in\left[0, t_{1}\right]$; we can see that $d x(t) / d t \geq-\beta(v(t) x(t) / T(t))$, and clearly $y(t)<T(t)$, for $t \in\left[0, t_{1}\right]$; these observations imply that, for $t \in\left[0, t_{1}\right]$, we have $d x(t) / d t \geq-\beta(v(t) x(t) / y(t))$.

Hence,

$$
x\left(t_{1}\right) \geq x(0) e^{-\int_{0}^{t_{1}}(\beta v(s) / y(s)) d s}>0,
$$

which contradicts our assumption.

Following a similar approach, we can prove that all the variables of system (3) are positive.

In order to prove boundedness of the solutions of system (3), we consider the following function:

$$
\begin{aligned}
F(t)= & N c g e^{-k \tau} x(t)+N c g y(t+\tau)+\frac{c g}{2} v(t+\tau) \\
& +\frac{c q}{2} w(t+\tau)+N g p z(t+\tau)
\end{aligned}
$$

From (3), we have

$$
\begin{aligned}
& \frac{d F(t)}{d t}=N c g e^{-k \tau}\left(r x(t)-r x(t) \frac{T(t)}{T_{m}}\right. \\
& \left.-\beta \frac{v(t) x(t)}{T(t)}\right)+N c g\left(\beta e^{-k \tau} \frac{v(t) x(t)}{T(t)}\right. \\
& -a y(t+\tau)-p y(t+\tau) z(t+\tau)) \\
& +\frac{c g}{2}(a N y(t+\tau)-\delta v(t+\tau) \\
& -q v(t+\tau) w(t+\tau))+\frac{c q}{2}(g v(t+\tau) w(t+\tau) \\
& -h w(t+\tau))+N g p(c y(t+\tau) z(t+\tau) \\
& -b z(t+\tau)) ;
\end{aligned}
$$

since $0<T(t)<T_{m}, x(t)<T_{m},-x(t) T(t)<-x(t)$ for $t>$ 0 , it follows that

$$
\begin{aligned}
\frac{d F(t)}{d t} \leq & N c g e^{-k \tau} r T_{m}-N c g e^{-k \tau} \frac{r}{T_{m}} x(t) \\
& -\frac{a N c g}{2} y(t+\tau)-\frac{\delta c g}{2} v(t+\tau) \\
& -\frac{h c q}{2} w(t+\tau)-N g p b z(t+\tau) ;
\end{aligned}
$$

if we set $\varrho=\min \left(r / T_{m}, a / 2, \delta, h, b\right)$, we will have

$$
\frac{d F(t)}{d t} \leq N c g e^{-k \tau}-\varrho F(t) .
$$

Using Gronwall's Lemma, we have that $F(t)$ is bounded, which makes the variables $x(t), y(t), v(t), w(t)$, and $z(t)$ bounded, which makes us unsure that the solution exists globally.

The above results show that the components of the solution of system (3) are nonnegative for all $t \in[0, b)$. Hence, the boundedness of $T(t), v(t), w(t)$, and $z(t)$ on $[0, b)$ imply that $b=\infty$. This completes the proof of the theorem.

\section{Equilibrium Points and Their Stability}

First, we aim to find all possible equilibria points. It is clear that our model (3) has disease-free equilibrium $E_{0}=\left(T_{m}\right.$, $0,0,0,0)$. The second equilibrium $E_{1}=\left(x_{1}, y_{1}, v_{1}, 0,0\right)$ represents the no immune response equilibrium with

$$
\begin{aligned}
x_{1} & =\frac{T_{m} \beta a N}{\mathscr{R}_{0} \delta r}\left(\mathscr{R}_{1}-1\right), \\
y_{1} & =\frac{T_{m} \beta a N}{\mathscr{R}_{0} \delta r}\left(\mathscr{R}_{0}-1\right)\left(\mathscr{R}_{1}-1\right), \\
v_{1} & =\frac{(a N)^{2} \beta T_{m}}{\delta^{2} \mathscr{R}_{0} r}\left(\mathscr{R}_{0}-1\right)\left(\mathscr{R}_{1}-1\right) .
\end{aligned}
$$

This equilibrium exits only if $\mathscr{R}_{0}>1$ and $\mathscr{R}_{1}>1$ with

$$
\begin{aligned}
& \mathscr{R}_{0}=\frac{\beta e^{-k \tau} N}{\delta}, \\
& \mathscr{R}_{1}=\frac{\delta r \mathscr{R}_{0}+\beta a N}{\beta a N \mathscr{R}_{0}}=\left(\frac{r e^{-k \tau}}{a}+1\right) \frac{1}{\mathscr{R}_{0}} .
\end{aligned}
$$

We also have the equilibrium $E_{2}=\left(x_{2}, y_{2}, v_{2}, 0, z_{2}\right)$, which represents an equilibrium where CTL cells are the only adaptive immune response and B-cells are zero. To define such equilibrium, we introduce the following thresholds:

$$
\mathscr{R}^{\star}=\frac{T_{m} \delta c r}{4 \beta a N b} .
$$

If $\mathscr{R}^{\star} \geq 1$, then we define

$$
\begin{aligned}
& \mathscr{R}_{2}=\frac{c T_{m}}{2 b}\left(1+\sqrt{1-\frac{1}{\mathscr{R}^{\star}}}\right), \\
& \mathscr{R}_{z}=\mathscr{R}_{0}\left(1-\frac{1}{\mathscr{R}_{2}}\right) .
\end{aligned}
$$


If $\mathscr{R}_{2}>1$ and $\mathscr{R}_{z}>1$, then the coordinates of $E_{2}$ are given by

$$
\begin{aligned}
& x_{2}=\frac{b}{c}\left(\mathscr{R}_{2}-1\right), \\
& y_{2}=\frac{b}{c}, \\
& v_{2}=\frac{a N b}{\delta c}, \\
& z_{2}=\frac{a}{p}\left(\mathscr{R}_{z}-1\right) .
\end{aligned}
$$

Remark 2 . If $\mathscr{R}_{0}>1$, we can easily prove the following:

(1) The equilibrium $E_{1}$ exists if and only if

$$
1<\mathscr{R}_{0}<\frac{r e^{-k \tau}}{a}+1 .
$$

(2) $\mathscr{R}^{\star}>1$ is equivalent to

$$
1<\mathscr{R}_{0}<\frac{T_{m} c r e^{-k \tau}}{4 a b} .
$$

And if $\mathscr{R}_{2}>1$, this condition combined with the condition $\mathscr{R}_{z}>1$ could be simplified to

$$
1<\frac{\mathscr{R}_{2}}{\mathscr{R}_{2}-1}<\mathscr{R}_{0}<\frac{T_{m} c r e^{-k \tau}}{4 a b} .
$$

From the two previous assessments, the model could have two equilibria $E_{1}$ and $E_{2}$ at same time if

$$
1<\frac{\mathscr{R}_{2}}{\mathscr{R}_{2}-1}<\mathscr{R}_{0}<\min \left(\frac{T_{m} c r e^{-k \tau}}{4 a b}, \frac{r e^{-k \tau}}{a}+1\right) .
$$

Finally, it is easy to see that if $b / c \leq T_{m} / 2$ or $b / c \geq T_{m}$, then $\mathscr{R}_{2}>1$.

The third type of equilibrium, $E_{3}$, is characterised by no CTL cells response; that is, $z=0$. For this reason, we define the threshold by

$$
\mathscr{R}^{\diamond}=\frac{T_{m} g r}{4 \beta h} .
$$

If $\mathscr{R}^{\diamond} \geq 1$, we define $T_{l}$ and $T_{h}$ (with $T_{l} \leq T_{h}$ ) by

$$
\begin{aligned}
& T^{h}=\frac{T_{m}}{2}\left(1+\sqrt{1-\frac{1}{\mathscr{R}^{\diamond}}}\right), \\
& T^{l}=\frac{T_{m}}{2}\left(1-\sqrt{1-\frac{1}{\mathscr{R}^{\diamond}}}\right) .
\end{aligned}
$$

Hence, the coordinates of $E_{3}^{i}=\left(x_{3}^{i}, y_{3}^{i}, v_{3}, w_{3}^{i}, 0\right)$, with $i=$ $l, h$, are given by

$$
\begin{aligned}
x_{3}^{i} & =\frac{a g\left(T^{i}\right)^{2}}{a g T^{i}+\beta e^{-k \tau} h} \\
y_{3}^{i} & =\frac{\beta e^{-k \tau} h T^{i}}{a g T^{i}+\beta e^{-k \tau} h} \\
v_{3} & =\frac{h}{g} \\
w_{3}^{i} & =\frac{a N g}{q} \frac{\beta e^{-k \tau} T^{i}}{a g T^{i}+\beta e^{-k \tau} h}-\frac{\delta}{q} .
\end{aligned}
$$

Notice that the virus coordinate does not depend on $T^{i}$. However, $x_{3}^{i}, y_{3}^{i}$, and $w_{3}^{i}$ are increase functions with respect to $T^{i}$.

Notice that $w_{3}^{i}>0$ require that $\mathscr{R}_{0}>1$ and

$$
T^{i}>\frac{1}{\Phi} \frac{\mathscr{R}_{0}}{\mathscr{R}_{0}-1}
$$

with $\Phi$ given by

$$
\Phi=\frac{a N}{\delta} \cdot \frac{g}{h} .
$$

Remark 3. We consider the threshold $\mathscr{R}_{T}$ defined by

$$
\mathscr{R}_{T}=\frac{a N g b}{\delta h c}=\Phi \frac{b}{c},
$$

which represents the survival rate of the virus, with ignoring the antibody effect, $a N / \delta$ times $g / h$ the survival rate of the antibody, over the survival rate of the CTL cells $c / b$.

It is easy to see that

$$
\begin{gathered}
\mathscr{R}^{*}>\mathscr{R}^{\diamond} \Longleftrightarrow \\
\mathscr{R}_{T}<1 \\
\left(\text { resp. } \mathscr{R}^{*}<\mathscr{R}^{\diamond} \Longleftrightarrow \mathscr{R}_{T}>1\right) .
\end{gathered}
$$

Finally, we have the endemic equilibria, $E_{4}=\left(x_{4}\right.$, $\left.y_{4}, v_{4}, w_{4}, z_{4}\right)$, where all the coordinates are nonzero. Using the same condition as the previous case, if $\mathscr{R}^{\diamond} \geq 1$, then there are two distinct $E_{4}^{i}=\left(x_{4}^{i}, y_{4}, v_{4}, w_{4}, z_{4}^{i}\right)$, with $i=l, h$, with the coordinate given by

$$
\begin{aligned}
& x_{4}^{i}=T^{i}-\frac{b}{c}, \\
& y_{4}=\frac{b}{c}, \\
& v_{4}=\frac{h}{g},
\end{aligned}
$$




$$
\begin{aligned}
w^{4} & =\frac{\delta}{q}\left(\mathscr{R}_{T}-1\right), \\
z_{4}^{i} & =\frac{\beta e^{-k \tau} h c}{p b g T^{i}}\left(T^{i}-\frac{b}{c}\right)-\frac{a}{p},
\end{aligned}
$$

and $T^{l}$ and $T^{h}$ are defined in (24).

The endemic equilibria are characterised by two possible levels of the healthy cells and corresponding CTL cells. On the other hand, coordinates of the endemic equilibria are constant with respect to the rest of the variables. It is important to mention that the existence of these two endemic equilibria requires $\mathscr{R}^{\diamond} \geq 1$ for $T^{i}$ to be feasible, and $\mathscr{R}_{T}>1, T^{i}>$ $b / c$, and $\left(\beta h e^{-k \tau} / a g T^{i}\right)\left(T^{i} c / b-1\right)>1$.

3.1. The Stability Analysis. In this section, we investigate the condition of stability of each possible equilibria point. First, the Jacobian matrix of system (3) is given by

$$
\left(\begin{array}{ccccc}
r\left(1-\frac{2 x+y}{T_{m}}\right)-\frac{\beta v y}{(x+y)^{2}} & \frac{-r x}{T_{m}}+\frac{\beta v x}{(x+y)^{2}} & -\frac{\beta x}{x+y} & 0 & 0 \\
\beta e^{-k \tau} \frac{v y}{(x+y)^{2}} & -\beta e^{-k \tau} \frac{v x}{(x+y)^{2}}-a-p z & \beta e^{-k \tau} \frac{x}{x+y} & 0 & -p y \\
0 & a N & -\delta-q w & -q v & 0 \\
0 & 0 & g w & g v-h & 0 \\
0 & c z & 0 & 0 & c y-b
\end{array}\right)
$$

and we have the following results.

Proposition 4. The free-equilibrium point $E_{0}$ is locally asymptotically stable when $\mathscr{R}_{0}<1$ and unstable when $\mathscr{R}_{0}>1$.

Proof. The characteristic polynomial of the Jacobian matrix (31) at $E_{0}$ is given by

$$
\begin{aligned}
P_{E_{0}}(\lambda)= & (\lambda+r)(\lambda+b)(-\lambda-h) \\
& \cdot\left(\lambda^{2}+(a+\delta) \lambda+a \delta\left(1-\mathscr{R}_{0}\right)\right),
\end{aligned}
$$

and then the eigenvalues of the Jacobian matrix at $E_{f}$ are

$$
\begin{gathered}
-r,-b,-h, \frac{-1}{2}\left(a+\delta+\sqrt{(a+\delta)^{2}+4 a \delta\left(\mathscr{R}_{0}-1\right)}\right) \\
\frac{-1}{2}\left(a+\delta-\sqrt{(a+\delta)^{2}+4 a \delta\left(\mathscr{R}_{0}-1\right)}\right) .
\end{gathered}
$$

It is clear that the first four eigenvalues are negative. The fifth one is negative when $\mathscr{R}_{0}<1$. We conclude that the free-equilibrium point $E_{0}$ is locally asymptotically stable when $\mathscr{R}_{0}<1$ and unstable when $\mathscr{R}_{0}>1$.

Next result will give the condition of stability of the no immune response equilibrium $E_{1}=\left(x_{1}, y_{1}, v_{1}, 0,0\right)$, where its coordinates are defined in (14).

Theorem 5. (1) If $\mathscr{R}_{0}<1$, then the point $E_{1}$ does not exist.

(2) If $\mathscr{R}_{0}=1$, then $E_{1}=E_{0}$.

(3) If $1<\mathscr{R}_{0}<1+r e^{-k \tau} / a$, then $E_{1}$ is locally asymptotically stable if $\min \left(\mathscr{H}_{0},(g a N / h \delta) \mathscr{H}_{0}\right)<1$; it is unstable for $\min \left(\mathscr{H}_{0},(g a N / h \delta) \mathscr{H}_{0}\right)>1$, with

$$
\mathscr{H}_{0}=\frac{T_{m} \beta a N}{\mathscr{R}_{0} \delta r}\left(\mathscr{R}_{0}-1\right)\left(\mathscr{R}_{1}-1\right) \text {. }
$$

Proof. Since the positivity of $y_{2}$ and $z_{2}$ depends on the positive sign of $\mathscr{R}_{0}-1$, we conclude that $E_{1}$ does not exist if $\mathscr{R}_{0}<1$. Moreover, if $\mathscr{R}_{0}=1$, it is easy to say that $E_{1}=$ $E_{f}$.

Next, we investigate the case where $1<\mathscr{R}_{0}<1+r e^{-k \tau} / a$. Using the Jacobian matrix (31), the characteristic equation at $E_{1}$ is as follows:

$$
\begin{aligned}
P_{E_{1}} & (\lambda)=\left(c y_{1}-b-\lambda\right)\left(g v_{1}-h-\lambda\right)\left(\lambda^{3}+a_{1} \lambda^{2}+a_{2} \lambda\right. \\
& \left.+a_{3}\right) \\
a_{1} & =\beta e^{-k \tau} T_{v} x_{1}+\delta+\beta T_{v} y_{1}+\frac{2 x_{1}+y_{1}}{T_{m}} r-r, \\
= & \beta e^{-k \tau} T_{v} x_{1}+\delta+\beta T_{v} y_{1}+\frac{\beta a N}{R_{0} \delta r}\left[\left(\frac{r e^{-k \tau}}{a}+1\right) \frac{1}{R_{0}}\right. \\
& -1]\left(R_{0}+1\right) . \\
a_{2} & =\beta e^{-k \tau} T_{v} x_{1}\left(\delta+\frac{2 x_{1}+y_{1}}{T_{m}} r-r+\beta T_{v} y_{1}\right) \\
& +\left(\frac{2 x_{1}+y_{1}}{T_{m}} r-r+\beta T_{v} y_{1}\right)(a+\delta)-a N \beta e^{-k \tau} T_{x}, \\
& +a N \beta^{2} e^{-k \tau} T_{v} y_{1} T_{x}, \\
a_{3} & =\left(\frac{\beta a N}{R_{0} \delta r}\left[\left(\frac{r e^{-k \tau}}{a}+1\right) \frac{1}{R_{0}}-1\right]\left(R_{0}+1\right)\right. \\
& \left.\beta T_{v} y_{1}\right)\left[\delta \beta e^{-k \tau} T_{v} x_{1}+a \delta+a N \beta e^{-k \tau} T_{x}\right]
\end{aligned}
$$


with

$$
\begin{aligned}
T_{v} & =\frac{v_{1}}{\left(x_{1}+y_{1}\right)^{2}}, \\
T_{x} & =\frac{x_{1}}{\left(x_{1}+y_{1}\right)} .
\end{aligned}
$$

Using the form of $v_{1}$ and $y_{1}$ given in (14), the two first eigenvalues $g v_{1}-h$ and $c y_{1}-b$ are negative (resp.) if and only if $(g a N / h \delta) \mathscr{H}_{0}<1$ and $\mathscr{H}_{0}<1$ (resp.).

On the other hand, from the Routh-Hurwitz theorem, the other eigenvalues of the above matrix have a negative real part when $1<\mathscr{R}_{0}<1+r e^{-k \tau} / a$.

Remark 6. (i) If $h \delta / a N g>1$, the condition $\min \left(\mathscr{H}_{0}\right.$, $\left.(g a N / h \delta) \mathscr{H}_{0}\right)<1$ can be replaced by $\mathscr{H}_{0}<1<h \delta / a N g$.

(ii) As the delay $\tau$ increases, by the inequality $1<\mathscr{R}_{0}<$ $1+r e^{-k \tau} / a$, the quantity $\mathscr{R}_{0}$ will be a bit bigger than one.

Next, we study the condition of local stability of the equilibrium $E_{2}$.

Theorem 7. Assume that $\mathscr{R}_{0}>1$; then the following applies:

(1) If $\mathscr{R}_{2} \leq 1$, then $E_{2}$ does not exist.

(2) If $\mathscr{R}_{2}>1$

(a) If $\mathscr{R}_{2} /\left(\mathscr{R}_{2}-1\right)>\mathscr{R}_{0}$, then $E_{2}$ does not exist.

(b) If $\mathscr{R}_{2} /\left(\mathscr{R}_{2}-1\right)=\mathscr{R}_{0}$, then $E_{2}=E_{1}$.

(c) If $\mathscr{R}_{2} /\left(\mathscr{R}_{2}-1\right)<\mathscr{R}_{0}<T_{m} c r e^{k \tau} / 4 a b$

(i) If $\Phi<1$, then $E_{2}$ is locally asymptotically stable.

(ii) If $\Phi>1$, then $E_{2}$ is unstable, where $\Phi$ is defined in (27).

Proof. We can easily notice that $\mathscr{R}_{2} \leq 1$ and then $\mathscr{R}_{z} \leq 0$, and then $x_{2}<0$ and $z_{2}<0$, which means that $E_{2}$ does not exist.

On the other hand, if $\mathscr{R}_{2}>1$ and $1<\mathscr{R}_{0}<\mathscr{R}_{2} /\left(\mathscr{R}_{2}-\right.$ 1), then $z_{2}<0$, and if $\mathscr{R}_{2}>1$ and $1<\mathscr{R}_{0}=\mathscr{R}_{2} /\left(\mathscr{R}_{2}-1\right)$, then $R_{z}=1$ and then $z_{2}=w_{2}=0$ and $E_{2}=E_{1}$.

Assume that $\mathscr{R}_{2}>1$ and condition (22) holds. From (31), the characteristic equation at $E_{2}$ is given by

$$
\begin{aligned}
& P_{E_{2}}(\lambda) \\
& \quad=\left(g v_{2}-h-\lambda\right)\left(\lambda^{4}+b_{1} \lambda^{3}+b_{2} \lambda^{2}+b_{3} \lambda+b_{4}\right),
\end{aligned}
$$

where

$$
\begin{aligned}
b_{1} & =\delta+\beta e^{-k \tau} T_{v} x_{2}+a+p z_{2}+\beta T_{v} y_{2}+\frac{2 x_{2}+y_{2}}{T_{m}} r \\
& -r
\end{aligned}
$$

$$
\begin{aligned}
b_{2}= & \delta \beta e^{-k \tau} T_{v} x_{2}+a \delta+p \delta z_{2}-a N \beta e^{-k \tau} T_{x}+p y_{2} \\
& +\left(\beta T_{v} y_{2}+\frac{2 x_{2}+y_{2}}{T_{m}} r-r\right) \\
& \cdot\left(\delta+\beta e^{-k \tau} T_{v} x_{2}+a+p z_{2}\right), \\
b_{3}= & \left(b-c y_{2}\right) \\
& \cdot\left(\delta \beta e^{-k \tau} T_{v} x_{2}+a \delta+p \delta z_{2}-a N \beta e^{-k \tau} T_{x}\right) \\
& +p y_{2} c z_{2} \delta+\left(\beta T_{v} y_{2}+\frac{2 x_{2}+y_{2}}{T_{m}} r-r\right) \\
& \cdot\left(\delta \beta e^{-k \tau} T_{v} x_{2}+a \delta+p \delta z_{2}-a N \beta e^{-k \tau} T_{x}+p y_{2}\right), \\
b_{4}= & \left(\beta T_{v} y_{2}+\frac{2 x_{2}+y_{2}}{T_{m}} r-r\right)\left(c y_{2}-b\right) \\
& \cdot\left(\delta \beta e^{-k \tau} T_{v} x_{2}+a \delta+p \delta z_{2}-a N \beta e^{-k \tau} T_{x}\right) \\
& -\left(\beta T_{v} y_{2} \frac{2 x_{2}+y_{2}}{T_{m}} r-r\right) p y_{2} c z_{2} \delta,
\end{aligned}
$$

with

$$
\begin{aligned}
& T_{v}=\frac{v_{1}}{\left(x_{1}+y_{1}\right)^{2}}, \\
& T_{x}=\frac{x_{1}}{\left(x_{1}+y_{1}\right)} .
\end{aligned}
$$

It is clear that $g v_{2}-h=h(\Phi-1)$ is an eigenvalue of $J_{E_{2}}$. The sign of this eigenvalue is negative if $\Phi<1$, positive if $\Phi>1$, and zero when $\Phi=1$. On the other hand, from the RouthHurwitz theorem applied to the fourth-order polynomial in the characteristic equation, the other eigenvalues of the above matrix have negative real parts when $\Phi<1$. Consequently, if $\mathscr{R}_{2}>1$ and $\mathscr{R}_{2} /\left(\mathscr{R}_{2}-1\right)<\mathscr{R}_{0}<T_{m} c r e^{k \tau} / 4 a b$, then $E_{2}$ is unstable when $\Phi>1$ and locally asymptotically stable when $\Phi<1$.

Now, we aim to find the condition of local stability of the equilibrium $E_{3}^{h}$; we have the following result.

Theorem 8. Assume that $\mathscr{R}_{0}>1$ and $\mathscr{R}^{\diamond}>1$ :

(1) If $T^{h}<\left(\beta e^{-\tau k} h / a g\right)\left(1 /\left(\mathscr{R}_{0}-1\right)\right)$, then equilibria $E_{3}^{i}$ for $i=l, h$ do not exist and $E_{3}^{h}=E_{1}$ when $T^{h}=\left(\beta e^{-\tau k} h / a g\right)\left(1 /\left(\mathscr{R}_{0}-1\right)\right)$.

(2) If $\left(\beta e^{-\tau k} h / a g\right)\left(1 /\left(\mathscr{R}_{0}-1\right)\right)<T^{h}<\left(1 / \mathscr{R}_{0}\right)(g N / \delta c h+$ $\left.\mathscr{R}_{0} / c\right)$, then $E_{3}^{h}$ are locally asymptotically stable.

(3) If $T^{h}>\max \left(\left(\beta e^{-\tau k} h / a g\right)\left(1 /\left(\mathscr{R}_{0}-1\right)\right),\left(1 / \mathscr{R}_{0}\right)(g N /\right.$ $\left.\left.\delta c h+\mathscr{R}_{0} / c\right)\right)$, then $E_{3}$ is unstable.

Proof. It is easy to see that if $T^{h}<\left(1 / \mathscr{R}_{0}\right)\left(\left(g+\beta h e^{-\tau k}\right) / a g\right)$, then the equilibrium $E_{3}^{h}$ does not exist and if $T^{h}=$ $\left(\beta e^{-\tau k} h / a g\right)\left(1 /\left(\mathscr{R}_{0}-1\right)\right)$ the two points $E_{3}^{h}$ and $E_{1}$ coincide. 
If $T^{h}>\left(\beta e^{-\tau k} h / a g\right)\left(1 /\left(\mathscr{R}_{0}-1\right)\right)$, using the Jacobian matrix (31), we get the following characteristic equation at $E_{3}^{i}$ :

$$
P_{E_{3}^{i}}(\lambda)=\left(c y_{3}^{i}-b-\lambda\right)\left(\lambda^{4}+c_{1} \lambda^{3}+c_{2} \lambda^{2}+c_{3} \lambda+c_{4}\right),
$$

where

$$
\begin{aligned}
& c_{1}=\beta e^{-k \tau} T_{v} x_{3}^{i}+a+\delta+\frac{2 x_{3}^{i}+y_{3}^{i}}{T_{m}} r-r+\beta T_{v} y_{3}^{i} \\
& +q w_{3}^{i} \\
& c_{2}=q v_{3} g w_{3}^{i}+\left(\delta+q w_{3}^{i}\right)\left(h-g v_{3}\right)+\left(\beta e^{-k \tau} T_{v} x_{3}^{i}\right. \\
& +a)\left(\delta+q w_{3}^{i}-g v_{3}+h+a N \beta e^{-k \tau} T_{x}\right) \\
& +\left(\frac{2 x_{3}^{i}+y_{3}^{i}}{T_{m}} r-r+\beta T_{v} y_{3}^{i}\right)\left(\beta e^{-k \tau} T_{v} x_{3}^{i}+a-q w_{3}^{i}\right. \\
& \left.+g v_{3}-\delta-h\right)-\beta e^{-k \tau} T_{v} y_{3}^{i}\left(-\frac{r x_{1}^{i}}{T_{m}}+\beta T_{v} x_{3}^{i}\right), \\
& c_{3}=\left(-\beta e^{-k \tau} T_{v} x_{3}^{i}-a\right)\left(\delta+q w_{3}^{i}\right)\left(g v_{3}-h\right) \\
& +\left(\beta e^{-k \tau} T_{v} x_{3}^{i}+a\right) q v_{3} g w_{3}^{i}+a N \beta e^{-k \tau} T_{x}\left(g v_{3}-h\right) \\
& +\left(-\frac{2 x_{3}^{i}+y_{3}^{i}}{T_{m}} r+r-\beta T_{v} y_{3}^{i}\right)\left(-\beta e^{-k \tau} T_{v} x_{3}^{i}-a\right)(\delta \\
& \left.+q w_{3}^{i}-g v_{3}+h+a N \beta e^{-k \tau} T_{x}\right)+\left(-\frac{2 x_{3}^{i}+y_{3}^{i}}{T_{m}} r\right. \\
& \left.+r-\beta T_{v} y_{3}^{i}\right)\left(\delta+q w_{3}^{i}\right)\left(g v_{3}-h\right)+\left(\frac{2 x_{3}^{i}+y_{3}^{i}}{T_{m}} r\right. \\
& \left.-r+\beta T_{v} y_{3}^{i}\right) q v_{3} g w_{3}^{i}+\beta e^{-k \tau} T_{v} y_{3}^{i}\left(\beta T_{x} a N\right. \\
& +\left(g v_{3}-h\right)\left(-\frac{r x_{1}^{i}}{T_{m}}+\beta T_{v} x_{3}^{i}\right) \\
& \left.+\left(-\frac{r x_{1}}{T_{m}}+\beta T_{v} x_{3}^{i}\right)\left(\delta+q w_{3}^{i}\right)\right), \\
& c_{4}=\left(-\frac{2 x_{3}^{i}+y_{3}^{i}}{T_{m}} r+r-\beta T_{v} y_{3}^{i}\right)\left(-\beta e^{-k \tau} T_{v} x_{3}^{i}-a\right) \\
& \cdot\left(\left(\delta+q w_{3}^{i}\right)\left(h-g v_{3}\right)+q v_{3} g w_{3}^{i}\right)+\left(-\frac{r x_{3}^{i}}{T_{m}}\right. \\
& \left.+\beta T_{v} x_{3}^{i}\right)\left(\delta+q w_{3}^{i}\right) g v_{3} \beta e^{-k \tau} T_{v} y_{3}^{i} \\
& -\beta e^{-k \tau} T_{v} y_{3}^{i}\left(q v_{3} g w_{3}^{i}\left(-\frac{r x_{3}^{i}}{T_{m}}+\beta T_{v} x_{3}^{i}\right)\right. \\
& \left.+\left(g v_{3}-h\right) \beta T_{x} a N\right) \text {, }
\end{aligned}
$$

with

$$
\begin{aligned}
& T_{v}=\frac{v_{3}}{\left(x_{3}^{i}+y_{3}^{i}\right)^{2}}, \\
& T_{x}=\frac{x_{3}^{i}}{\left(x_{3}^{i}+y_{3}^{i}\right)} .
\end{aligned}
$$

It is clear that $c y_{3}^{h}-b=b\left(c \beta h e^{-k \tau} T^{h} / b\left(g+\beta e^{-k \tau} h\right)-\right.$ $1)$ is an eigenvalue of $J_{E_{3}^{h}}$. The sign of this eigenvalue is negative if $T^{h}<b\left(g+\beta e^{-k \tau} h\right) / c \beta h e^{-k \tau}$, which is equivalent to $T^{h}<\left(1 / \mathscr{R}_{0}\right)\left(g N / \delta c h+\mathscr{R}_{0} / c\right)$. The sign of this eigenvalue is positive if $T^{h}>\left(1 / \mathscr{R}_{0}\right)\left(g N / \delta c h+\mathscr{R}_{0} / c\right)$, which will give, with $T^{h}>\left(1 / \mathscr{R}_{0}\right)\left(\left(g+\beta h e^{-\tau k}\right) / a g\right)$, the condition of instability of the theorem.

On the other hand, from the Routh-Hurwitz theorem, the other eigenvalues of the above matrix have a negative real part when $T^{h}<b\left(g+\beta e^{-k \tau} h\right) / c \beta h e^{-k \tau}$.

Consequently, if $\left(\beta e^{-\tau k} h / a g\right)\left(1 /\left(\mathscr{R}_{0}-1\right)\right)<T^{h}<$ $\left(1 / \mathscr{R}_{0}\right)\left(g N / \delta c h+\mathscr{R}_{0} / c\right)$, then $E_{3}^{h}$ is locally asymptotically stable.

Theorem 9. (1) If $\Phi<1$ or $H_{i}^{w, z}<1$, then the point $E_{4}^{i}$ with $i=l, h$ does not exist. Moreover, $E_{4}^{i}=E_{2}$ when $\Phi=$ 1 and $E_{4}^{i}=E_{2}$ when $H_{i}^{w, z}=1$.

(2) If $\Phi>1$ and $H_{i}^{w, z}>1$, then $E_{4}^{i}$ is locally asymptotically stable.

Here

$$
H_{i}^{w, z}=\frac{c \beta e^{-k \tau} h T^{i}}{b\left(g+\beta e^{-k \tau} h\right)} ; \quad i=l, h .
$$

\section{The Optimal Control Therapy Analysis}

In this section, we consider the optimal control of the HBV drug therapy; as we mentioned previously, the therapy has an antiviral effect by reducing the viral production rate and blocking the shedding and bending of the virus to the uninfected cells. For this purpose, we consider the controlled version of system (3) defined as follows:

$$
\begin{aligned}
\frac{d x}{d t}= & r x(t)\left(1-\frac{T(t)}{T_{m}}\right)-\beta\left(1-u_{1}(t)\right) \frac{v(t) x(t)}{T(t)}, \\
\frac{d y}{d t}= & \beta e^{-k \tau}\left(1-u_{1}(t)\right) \frac{v(t-\tau) x(t-\tau)}{T(t-\tau)}-a y(t) \\
& -p y(t) z(t) \\
\frac{d v}{d t}= & \left(1-u_{2}(t)\right) a N y(t)-\delta v(t)-q v(t) w(t), \\
\frac{d w}{d t}= & g v(t) w(t)-h w(t) \\
\frac{d z}{d t}= & c y(t) z(t)-b z(t) .
\end{aligned}
$$


The optimization problem that we consider is to maximize the following objective functional:

$$
\begin{gathered}
J\left(u_{1}, u_{2}\right)=\int_{0}^{t_{f}}\{x(t)+z(t)+w(t) \\
\left.-\left[\frac{A_{1}}{2} u_{1}^{2}(t)+\frac{A_{2}}{2} u_{2}^{2}(t)\right]\right\} d t
\end{gathered}
$$

where $t_{f}$ stands for the time period of treatment. The two positive constants $A_{1}$ and $A_{2}$ are the weight for the treatment. It is legitimate to assume that two control functions, $u_{1}(t)$ and $u_{2}(t)$, are bounded and Lebesgue integrable. These assumptions align with the fact that the drug has a limited dosage and time to use.

The goal is to decrease the viral load while increasing the number of the uninfected cells and maximizing the immune responses. This should be done with minimizing the cost of treatment. We can achieve this goal by maximizing the objective functional defined in (45), which means finding the optimal control pair $\left(u_{1}^{*}, u_{2}^{*}\right)$ such that

$$
J\left(u_{1}^{*}, u_{2}^{*}\right)=\max \left\{J\left(u_{1}, u_{2}\right):\left(u_{1}, u_{2}\right) \in U\right\},
$$

where $U$ is the control set defined by

$$
\begin{aligned}
U & =\left\{\left(u_{1}(t), u_{2}(t)\right): u_{i}(t) \text { measurable, } 0 \leq u_{i}(t)\right. \\
& \left.\leq 1, t \in\left[0, t_{f}\right], i=1,2\right\} .
\end{aligned}
$$

First, we need to ensure the existence of the optimal control pair. Using the results in Fleming and Rishel [33] and Lukes [34], we have the following theorem.

Theorem 10. There exists an optimal control pair $\left(u_{1}^{*}, u_{2}^{*}\right) \in$ $U$ such that

$$
J\left(u_{1}^{*}, u_{2}^{*}\right)=\max _{\left(u_{1}, u_{2}\right) \in U} J\left(u_{1}, u_{2}\right) .
$$

The proof of this result is omitted since it is similar to the one in Tridane et al. [16].

Next, via Pontryagin's Minimum Principle [35], we give the necessary conditions for an optimal control problem. We convert solving our optimization problem into maximizing the Hamiltonian $H \equiv H\left(t, x, y, v, z, w, x_{\tau}, v_{\tau}, u_{1}, u_{2}, \lambda_{i}\right)$ point-wisely with respect to $u_{1}$ and $u_{2}$ as follows:

$$
\begin{aligned}
H= & \frac{A_{1}}{2} u_{1}(t)^{2}+\frac{A_{2}}{2} u_{2}(t)^{2}-x(t)-z(t)-w(t) \\
& +\sum_{i=0}^{5} \lambda_{i} f_{i}
\end{aligned}
$$

with

$$
\begin{aligned}
f_{1}= & r x(t)\left(1-\frac{T(t)}{T_{m}}\right)-\beta\left(1-u_{1}(t)\right) \frac{v(t) x(t)}{T(t)}, \\
f_{2}= & \beta e^{-k \tau}\left(1-u_{1}(t)\right) \frac{v(t-\tau) x(t-\tau)}{T(t-\tau)}-a y(t) \\
& -p y(t) z(t) \\
f_{3}= & \left(1-u_{2}(t)\right) a N y(t)-\delta v(t)-q v(t) w(t), \\
f_{4}= & g v(t) w(t)-h w(t) \\
f_{5}= & c y(t) z(t)-b z(t) .
\end{aligned}
$$

And $\lambda_{i}, i=1,2,3,4,5$, are the adjoint functions to be determined. By applying Pontryagin's Minimum Principle in the case system with delay [35], we have the following theorem.

Theorem 11. Given optimal controls $u_{1}^{*}, u_{2}^{*}$ and solutions $x^{*}$, $y^{*}, v^{*}, z^{*}$, and $w^{*}$ of the corresponding state system (3), there exist adjoint variables, $\lambda_{1}, \lambda_{2}, \lambda_{3}, \lambda_{4}$, and $\lambda_{5}$ satisfying the equations

$$
\begin{aligned}
& \frac{d \lambda_{1}(t)}{d t}=1-\lambda_{1}(t)\left[r\left(1-\frac{T^{*}(t)}{T_{m}}\right)-\frac{r x^{*}(t)}{T_{m}}\right. \\
& \left.-\left(1-u_{1}^{*}(t)\right) \beta v^{*}(t) \frac{y^{*}(t)}{T^{* 2}}\right]-\chi_{\left[0, t_{f}-\tau\right]}(t) \lambda_{2}(t \\
& +\tau)\left(u_{1}^{*}(t+\tau)-1\right) \beta e^{-k \tau} v^{*}(t) \frac{y^{*}(t)}{T^{* 2}(t)}, \\
& \frac{d \lambda_{2}(t)}{d t}=\lambda_{1}(t)\left(\frac{r x^{*}(t)}{T_{m}}\right. \\
& \left.-\left(1-u_{1}^{*}(t)\right) \beta v^{*}(t) \frac{x^{*}(t)}{T^{* 2}}\right)+\lambda_{2}(t)(a+p z) \\
& -\lambda_{3}(t)\left(1-u_{2}^{*}(t)\right) a N-c z^{*}(t) \lambda_{5}(t) \\
& -\chi_{\left[0, t_{f}-\tau\right]}(t) \lambda_{2}(t+\tau)\left(u_{1}^{*}(t+\tau)-1\right) \beta e^{-k \tau} v^{*}(t) \\
& \frac{x^{*}(t)}{T^{* 2}(t)} \\
& \frac{d \lambda_{3}(t)}{d t}=\lambda_{1}(t)\left[\beta\left(1-u_{1}^{*}(t)\right) \frac{x^{*}(t)}{T^{*}(t)}\right]+\lambda_{3}(t)(\delta \\
& +q w(t))-\lambda_{4}(t) g w^{*}(t)+\chi_{\left[0, t_{f}-\tau\right]}(t) \lambda_{2}(t+\tau) \\
& \cdot\left[\beta e^{-k \tau}\left(u_{1}^{*}(t+\tau)-1\right) \frac{x^{*}(t)}{T^{*}(t)}\right], \\
& \frac{d \lambda_{4}(t)}{d t}=1+\lambda_{3}(t) q v^{*}(t)+\lambda_{4}(t)\left[h-g v^{*}(t)\right], \\
& \frac{d \lambda_{5}(t)}{d t}=1+\lambda_{2}(t) p y^{*}(t)+\lambda_{5}(t)\left[b-c y^{*}(t)\right],
\end{aligned}
$$

with $\chi$ being an indicator function and $T^{*}(t)=x^{*}(t)+$ $y^{*}(t)$ also the transversality conditions 


$$
\lambda_{i}\left(t_{f}\right)=0, \quad i=1, \ldots, 5
$$

$$
\begin{aligned}
& u_{1}^{*}=\min \left(1, \max \left(0, \frac{\beta}{A_{1}}\left[\lambda_{2}(t) e^{-k \tau} \frac{v^{*}(t-\tau) x^{*}(t-\tau)}{T^{*}(t-\tau)}-\lambda_{1}(t) \frac{v^{*}(t) x^{*}(t)}{T^{*}}\right]\right)\right) \\
& u_{2}^{*}=\min \left(1, \max \left(0, \frac{1}{A_{2}} \lambda_{3}(t) a N y^{*}(t)\right)\right) .
\end{aligned}
$$

\section{Numerical Simulations}

In order to solve our optimization system, we use a numerical schema based on the forward and backward finite difference approximation. This schema was originally presented in the case of ODE system in [36], used similarly by [37] and enhanced for delay differential equation system [38-40].

We consider the step size $h>0$ and $(n, m) \in \mathbb{N}^{2}$ with $\tau=m h$ and $t_{f}-t_{0}=n h$. We take $m$ knots to left of $t_{0}$ and right of $t_{f}$, to get the following partition:

$$
\begin{aligned}
\Delta & =\left(t_{-m}=-\tau<\cdots<t_{-1}<t_{0}=0<t_{1}<\cdots<t_{n}=t_{f}\right. \\
& \left.<t_{n+1}<\cdots<t_{n+m}\right)
\end{aligned}
$$

which gives $t_{i}=t_{0}+i h(-m \leq i \leq n+m)$. The state and the adjoint variables are $x(t), y(t), v(t), w(t), z(t), \lambda_{1}(t), \lambda_{2}(t)$, $\lambda_{3}(t), \lambda_{4}(t)$, and $\lambda_{5}(t)$ and the controls are $u_{1}(t), u_{2}(t)$ in terms of nodal points $x_{i}, y_{i}, v_{i}, w_{i}, z_{i}, \lambda_{1}^{i}, \lambda_{2}^{i}, \lambda_{3}^{i}, \lambda_{4}^{i}, \lambda_{5}^{i}, u_{1}^{i}$, and $u_{2}^{i}$. By combining the forward and backward difference approximation, we get Algorithm 1.

For the simulation, we use the parameter values given in Table 1 .

As the parameters having been chosen from different references (see Table 1), we use in our numerical simulations a set of parameters that are within the range of the estimation of these references; that is, $r=1, T_{m}=2 \times 10^{11}, \beta=0.0018$, $k=1.1 \times 10^{-2}, \tau=1, a=0.0693, N=480, \delta=0.693$, $q=0.01, p=0.001, c=4.4 \times 10^{-8}, b=0.5, q=10^{-10}$, $g=10^{-4}, h=0.1, A_{1}=250$, and $A_{2}=2500$.

First, we start our simulation by showing the effect of the delay on the dynamics of the different cells' population as well as the free virions particles. Figure 1 presents the time series of the uninfected cells, the infected cells, the free viruses, and the antibodies. The dashed curves represent the case with delay, while the solid curves show the case without delay. The delay has a clear effect on the dynamics of the early HBV infection by slowing down the overall time series by expending the time between the phases of each curve. However, there is no difference between the two cases as the time passes, which means that the time delay could have an effect on the time scale in planning the treatment period. However, the delay does not lead to periodic dynamics of the model. Hence, the delay cannot cause periodic oscillations.

The next illustrative simulation of the model aims to help in comparing the uninfected cells, the infected cells, the viral load, and the immune response with and without therapy.
Figure 2 shows an increase of the healthy hepatocytes (a) in the first three days, but it is clear that the therapy gives a substantial increase of healthy hepatocytes, with more than 200,000 cells, compared with the case without therapy.

We notice also that, in the absence of the therapy, the number of the infected hepatocytes (b) increases rapidly in the first four days, decreases within twenty days, and increases after 25 days, whereas, in the presence of treatment, the number of infected hepatocytes decreases asymptotically to an undetectable level. More precisely, the number of infected cells with control stabilises at 2.5482, while the number of infected cells without control reaches $2.264 \times 10^{5}$, which makes the drug therapy efficiency in blocking the new infections at $98.73 \%$.

In Figure 2, we see that the number of free virions (a) decreases rapidly towards an undetectable level after introducing the therapy. In fact, with control, the virus stabilises at 1.2112 while without control it reaches $9.768 \times$ $10^{6}$, which represents a perfect efficiency of the drug therapy in inhibiting the viral production (about 99.99\%).

Figure 3(b) shows the antibodies immune response as a function of time. Without the therapy, the antibody level shows relapse in count 50 days after the infection, before it persists over time. We can see clearly that the relapse of the antibody synchronised with the virus peak. On the other hand, the early therapy reduces the burden on the antibody as immune response is barely measured.

The optimal therapy protocol is represented by Figure 4. Each curve presents the optimal drug dosage efficiency and the drug timing during the time of therapy. The optimal therapy requires having a full dosage efficiency for both drugs; the efficiency should be for about 4 days for INF and about 2 weeks for NAs. After 4 days, the INF administration should be stopped and again retaken until it reaches 32\% efficiency. Later on, the efficiency can be dropped to less than $10 \%$. For the NAs drugs, after two weeks, the efficiency can be reduced to $50 \%$ and eventually dropped to $15 \%$ for the rest of the treatment duration.

\section{Conclusion}

In this paper, we investigated a mathematical model of the adaptive immune response of the early stage of HBV. The early stage is characterised by a delay in the infection process and a logistic growth of the healthy hepatocyte cells. The aim is to study the role of the two arms of the adaptive immune response, represented by the antibodies and the 
Step 1:

for $i=-m, \ldots, 0$, do:

$$
x_{i}=x_{0}, y_{i}=y_{0}, T_{i}=x_{0}+y_{0}, v_{i}=v_{0}, w_{i}=w_{0}, z_{i}=z_{0}, u_{1}^{i}=0, u_{2}^{i}=0 .
$$

end for

for $i=n, \ldots, n+m$, do:

end for

$\lambda_{1}^{i}=0, \lambda_{2}^{i}=0, \lambda_{3}^{i}=0, \lambda_{4}^{i}=0, \lambda_{5}^{i}=0$.

Step 2:

for $i=0, \ldots, n-1$, do

$$
\begin{aligned}
x_{i+1}= & x_{i}+h\left[r x_{i}\left(1-\frac{T_{i}}{T_{m}}\right)-\beta\left(1-u_{1}^{i}\right) \frac{v_{i} x_{i}}{T_{i}}\right] \\
y_{i+1}= & y_{i}+h\left[\beta e^{-k \tau}\left(1-u_{1}^{i}\right) v_{i-m} \frac{x_{i-m}}{T_{i-m}}-a y_{i}-p y_{i} z_{i}\right] \\
v_{i+1}= & v_{i}+h\left[\left(1-u_{2}^{i}\right) a N y_{i}-\delta v_{i}-q v_{i} w_{i}\right] \\
w_{i+1}= & w_{i}+h\left[g v_{i} w_{i}-h w_{i}\right] \\
z_{i+1}= & z_{i}+h\left[c y_{i} z_{i}-b z_{i}\right] \\
T_{i+1}= & x_{i+1}+y_{i+1}, \\
\lambda_{1}^{n-i-1}= & \lambda_{1}^{n-i}-h\left[1-\lambda_{1}^{n-i}\left(r\left(1-\frac{T_{i+1}}{T_{m}}\right)-r \frac{x_{i+1}}{T_{m}}-\left(1-u_{1}^{i}\right) \beta v_{i+1} \frac{y_{i+1}}{T_{i+1}}\right)-\chi_{\left[0, t_{f}-\tau\right]}\left(t_{n-i}\right) \lambda_{2}^{n-i+m}\left(u_{1}^{i+m}-1\right) \beta e^{-k \tau} v_{i+1} \frac{y_{i+1}}{T_{i+1}^{2}}\right] \\
\lambda_{2}^{n-i-1}= & \lambda_{2}^{n-i}-h\left[\lambda_{1}^{n-i}\left(r \frac{x_{i+1}}{T_{m}}-\left(1-u_{1}^{i}\right) \beta v_{i+1} \frac{x_{i+1}}{T_{i+1}^{2}}\right)+\lambda_{2}^{n-i}\left(a+p z_{i+1}\right)-\lambda_{3}^{n-i}\left(1-u_{2}^{i}\right) a N-c z_{i+1} \lambda_{5}^{n-i}\right] \\
& -\chi_{\left[0, t_{f}-\tau\right]}\left(t_{n-i}\right) \lambda_{2}^{n-i+m}\left(u_{1}^{i+m}-1\right) \beta e^{-k \tau} v_{i+1} \frac{x_{i+1}}{T_{i+1}^{2}}, \\
\lambda_{3}^{n-i-1}= & \lambda_{3}^{n-i}-h\left[\lambda_{1}^{n-i}\left(1-u_{1}^{i}\right) \beta \frac{x_{i+1}}{T_{i+1}}+\lambda_{3}^{n-i}\left(\delta+q w_{i+1}\right)-\lambda_{4}^{n-i} g w_{i+1}+\chi_{\left[0, t_{f}-\tau\right]}\left(t_{n-i}\right) \lambda_{2}^{n-i+m}\left(u_{1}^{i+m}-1\right) \beta e^{-k \tau} \frac{x_{i+1}}{T_{i+1}}\right] \\
\lambda_{4}^{n-i-1}= & \lambda_{4}^{n-i}-h\left[1+q \lambda_{3}^{n-i} v_{i+1}+\lambda_{4}^{n-i}\left(h-g v_{i+1}\right)\right] \\
\lambda_{5}^{n-i-1}= & \lambda_{4}^{n-i}-h\left[1+p \lambda_{2}^{n-i} y_{i+1}+\lambda_{5}^{n-i}\left(b-c y_{i+1}\right)\right] \\
R_{1}^{i+1}= & \left(\frac{\beta}{A_{1}}\right)\left(\lambda_{2}^{n-i-1} e^{-k \tau} v_{i-m+1} \frac{x_{i-m+1}}{T_{i-m+1}}-\lambda_{1}^{n-i-1} v_{i+1} \frac{x_{i+1}}{T_{i+1}}\right) \\
R_{2}^{i+1}= & \left(\frac{1}{A_{2}}\right) \lambda_{3}^{n-i-1} a N y_{i+1}, \\
u_{1}^{i+1}= & \min \left(1, \max \left(R_{1}^{i+1}, 0\right)\right) \\
u_{2}^{i+1}= & \min \left(1, \max \left(R_{2}^{i+1}, 0\right)\right), \\
f &
\end{aligned}
$$

\begin{tabular}{|c|c|c|c|}
\hline Parameters & Meaning & Value & References \\
\hline$r$ & Maximum hepatocyte growth rate & $\leq 1.0$ day $^{-1}$ & {$[23,24]$} \\
\hline$T_{m}$ & Hepatocyte carrying capacity & $2 \times 10^{11}$ cells & [25] \\
\hline$\beta^{m}$ & Rate of virion infection of hepatocytes & $3.6 \times 10^{-5}-1.8 \times 10^{-3}$ cells virion $^{-1}$ day $^{-1}$ & {$[26]$} \\
\hline$\tau$ & Time delay & 1 day & {$[27,28]$} \\
\hline$k$ & Normal death rate for hepatocytes & .0039 day $^{-1}$ & {$[27,28]$} \\
\hline$a$ & Infected hepatocyte death rate & $0.0693-0.00693 \mathrm{day}^{-1}$ & {$[25]$} \\
\hline$p$ & Clearance rate of infection & $7 \pm 1.7 \times 10^{-4} \mathrm{ml} /$ cell day $^{-1}$ & {$[29]$} \\
\hline$N$ & Number of free viruses produced by infected cells & 480 & {$[12,13]$} \\
\hline$\delta$ & Free virion half-life & $0.67 \mathrm{day}^{-1}$ & {$[30]$} \\
\hline$q$ & Neutralization rate of virion and antibodies & $10^{-10}-10^{-12} \mathrm{ml} \mathrm{day}^{-1}$ & {$[31]$} \\
\hline$g$ & Activation rate of B-cells & $1.38 \times 10^{-2}-10^{-4}$ day $^{-1}$ & {$[31]$} \\
\hline$h$ & Death rate of B-cells & $0.03-0.1$ day $^{-1}$ & {$[31]$} \\
\hline$c$ & Activation rate of CTL cells & $4.4 \pm 1.5 \times 10^{-7} \mathrm{ml} \mathrm{cell}^{-1}$ day $^{-1}$ & [29] \\
\hline$b$ & Death rate of CTL cells & 0.5 day $^{-1}$ & {$[32]$} \\
\hline
\end{tabular}

end for

Step 3:

for $i=1, \ldots, n$, write

$$
x^{*}\left(t_{i}\right)=x_{i}, y^{*}\left(t_{i}\right)=y_{i}, T^{*}\left(t_{i}\right)=T_{i}, v^{*}\left(t_{i}\right)=v_{i}, z^{*}\left(t_{i}\right)=z_{i}, w^{*}\left(t_{i}\right)=w_{i}, u_{1}^{*}\left(t_{i}\right)=u_{1}^{i}, u_{2}^{*}\left(t_{i}\right)=u_{2}^{i} .
$$

end for

\section{Algorithm 1}

TABLE 1: Parameters, their symbols, and default values used in Model (3). 


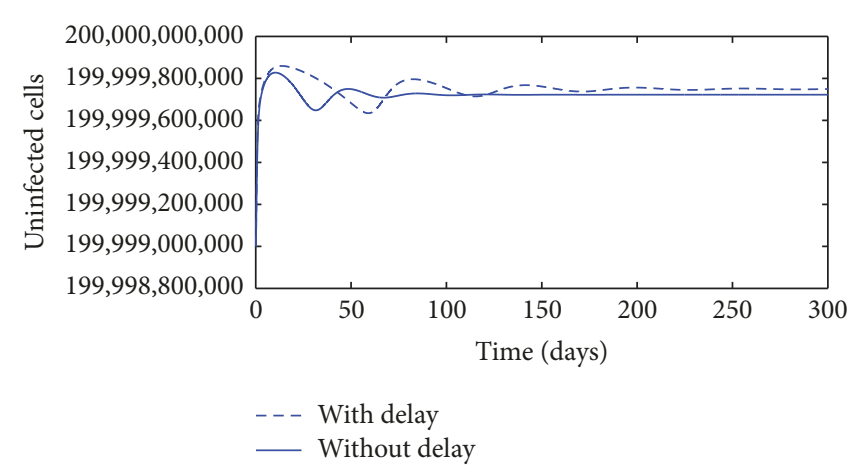

(a)

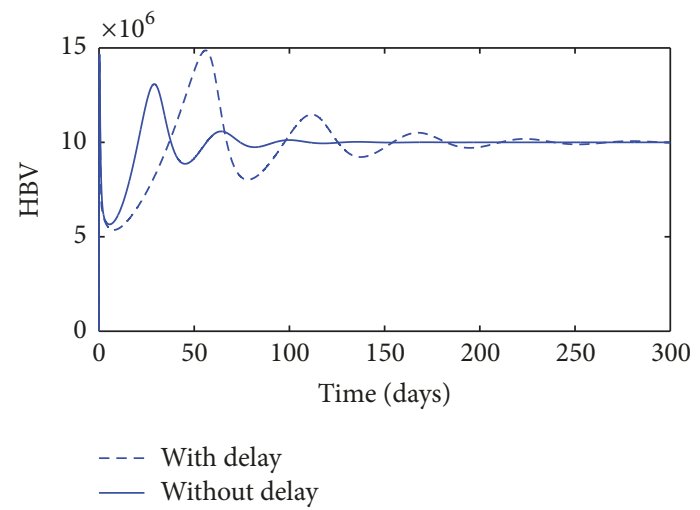

(c)

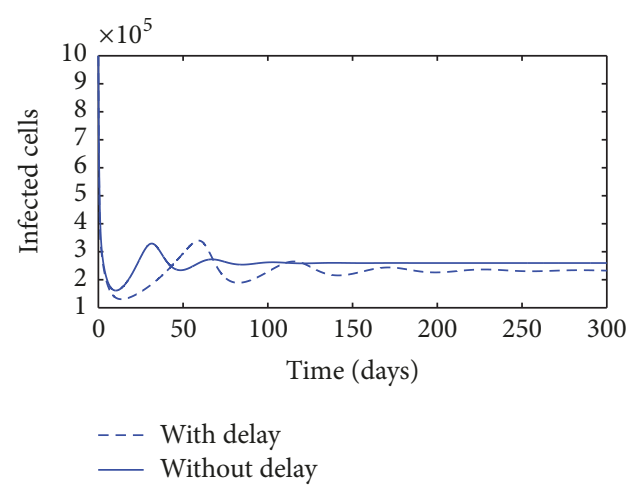

(b)

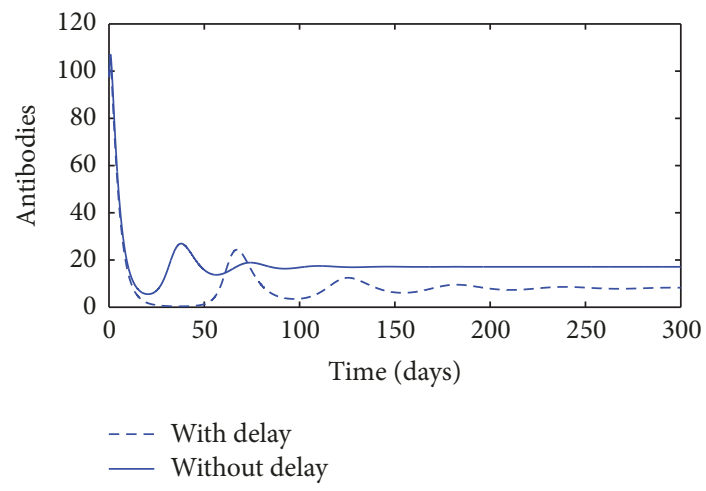

(d)

FIgure 1: The uninfected cells (a). The infected cells (b). The HBV (c). The antibody response (d).

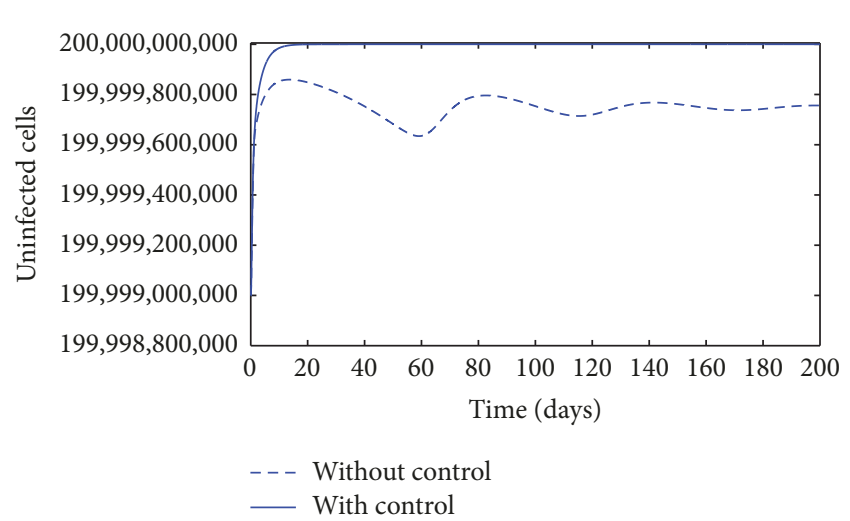

(a)

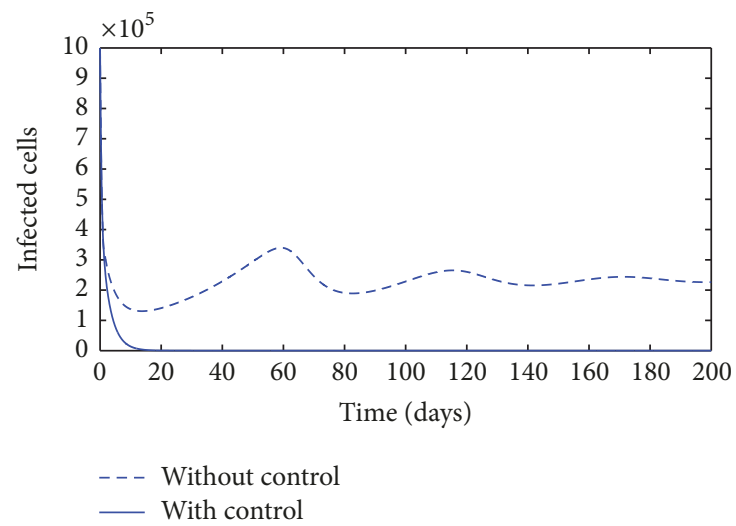

(b)

Figure 2: The uninfected cells as a function of time (a). The infected cells as a function of time (b).

CTL cells, in the progress of the HBV infection as the virus gains ground and becomes widespread. Our study showed the possibility of several outcomes depending on many thresholds, which led us to find the conditions of existence of four possible equilibria and investigate their local stability. The stability analysis of these equilibria was very involving and required rigorous calculations. Our mathematical analysis and numerical simulations show that the delay has the effect of slowing down the progress of the disease but does not lead to oscillatory behavior of the dynamics.
As a result of this finding, our next goal was to find the possibility of introducing the actual therapy, which includes standard interferon- $\alpha$ and nucleoside analogues. For this purpose, we investigated the optimal control of this therapy via the proposed model. The implementation of such therapy in the early stage instead of the acute stage of HBV infection could be helpful in reducing the burden of the disease. The optimal therapy aims to increase the efficacy of the drug while keeping the healthy hepatocyte cells at the normal level and enhancing the immune response. To solve this problem, 


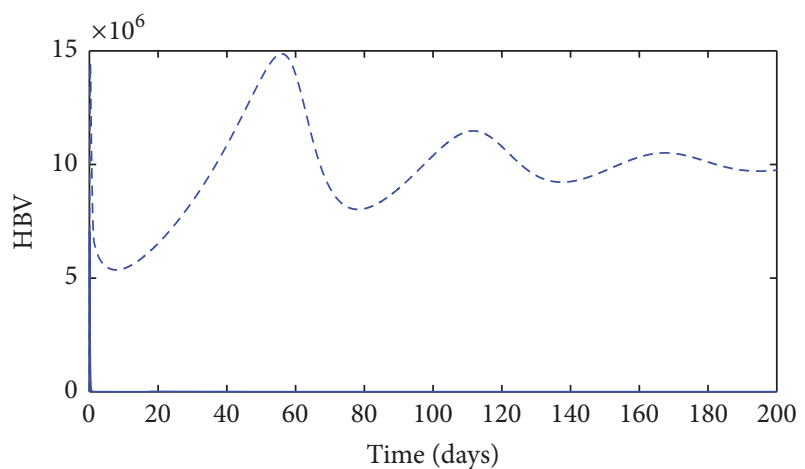

- - - Without control With control

(a)

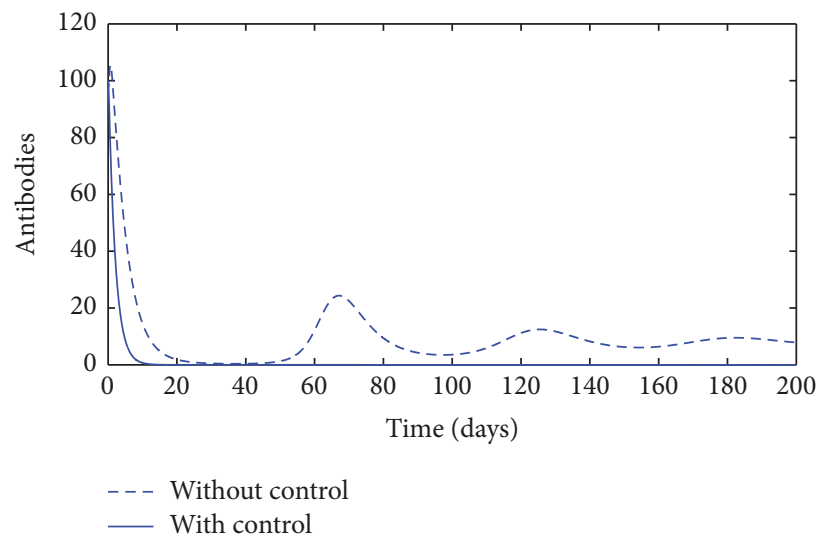

(b)

Figure 3: The HBV as a function of time (a). The antibody response as a function of time (b).

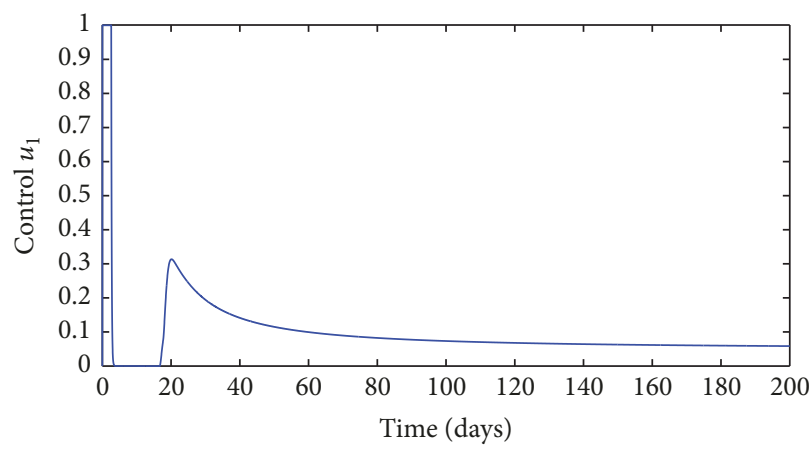

(a)

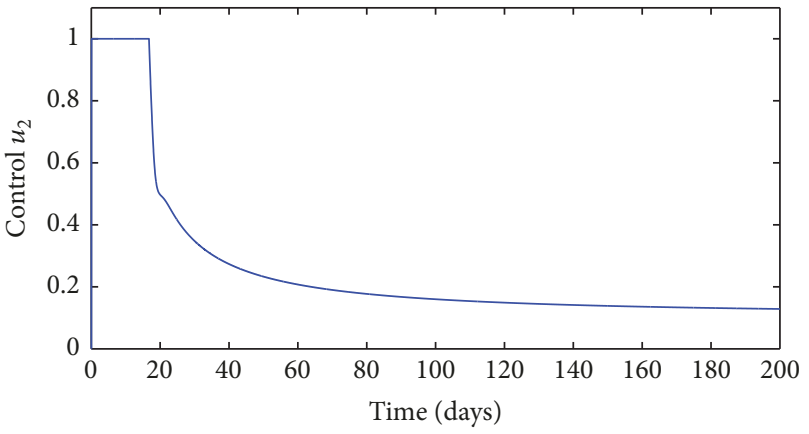

(b)

FIGURE 4: The optimal control $u_{1}$ (a) and the optimal control $u_{2}$ (b) versus time.

we used the standard techniques to prove the condition of existence of a solution and to find the optimality system. A well-known numerical method was used to solve the optimality system and to identify the best treatment strategy of HBV infection to block new infections and prevent viral production using drug therapy with minimum side effects on the immune response and the healthy hepatocyte cells.

Our numerical results show that the optimal treatment strategies should have high efficiency at the beginning of the therapy, about four days for INF and two weeks for NAs; the efficiency can be adjusted to $10 \%$ for INF and to $50 \%$ for NAs, and gradually to $15 \%$.

Since there is no clear guideline for the combination therapy in general [41] and for the early infection of HBV in particular, this work should serve as an initial step to consider an early combined use of IFN and NAs in HBV infection. Of course, more pharmacokinetic studies are needed to investigate the long time use of this therapy and the possible risk of treatment failure [8].

\section{Conflicts of Interest}

The authors declare that they have no conflicts of interest.

\section{References}

[1] C. Ferrari, "HBV and the immune response," Liver International, vol. 35, supplement 1, pp. 121-128, 2015.

[2] C. Ferrari, A. Penna, A. Bertoletti et al., "Cellular immune response to hepatitis $\mathrm{B}$ virus-encoded antigens in acute and chronic hepatitis B virus infection," The Journal of Immunology, vol. 145, no. 10, pp. 3442-3449, 1990.

[3] B. Rehermann and M. Nascimbeni, "Immunology of hepatitis B virus and hepatitis $C$ virus infection," Nature Reviews Immunology, vol. 5, no. 3, pp. 215-229, 2005.

[4] J. Waters, M. Pignatelli, S. Galpin, K. Ishihara, and H. C. Thomas, "Virus-neutralizing antibodies to hepatitis B virus: The nature of an immunogenic epitope on the S gene peptide," Journal of General Virology, vol. 67, no. 11, pp. 2467-2473, 1986.

[5] G. J. M. Webster, S. Reignat, M. K. Maini et al., "Incubation phase of acute hepatitis B in man: Dynamic of cellular immune mechanisms," Hepatology, vol. 32, no. 5, pp. 1117-1124, 2000.

[6] E. Loggi, N. Gamal, F. Bihl, M. Bernardi, and P. Andreone, "Adaptive response in hepatitis B virus infection," Journal of Viral Hepatitis, vol. 21, no. 5, pp. 305-313, 2014.

[7] D. R. Milich, M. Chen, F. Schödel, D. L. Peterson, J. E. Jones, and J. L. Hughes, "Role of B cells in antigen presentation of the hepatitis B core," Proceedings of the National Acadamy of 
Sciences of the United States of America, vol. 94, no. 26, pp. 14648-14653, 1997.

[8] S. Hagiwara, N. Nishida, and M. Kudo, "Antiviral therapy for chronic hepatitis B: Combination of nucleoside analogs and interferon," World Journal of Hepatology, vol. 7, no. 23, pp. 24272431, 2015.

[9] R.-N. Chien, C.-H. Lin, and Y.-F. Liaw, "The effect of lamivudine therapy in hepatic decompensation during acute exacerbation of chronic hepatitis B," Journal of Hepatology, vol. 38, no. 3, pp. 322-327, 2003.

[10] L. J. Sun, J. W. Yu, Y. H. Zhao, P. Kang, and S. C. Li, "Influential factors of prognosis in lamivudine treatment for patients with acute-on-chronic hepatitis B liver failure," Journal of Gastroenterology and Hepatology, vol. 25, no. 3, pp. 583-590, 2010.

[11] S. Eikenberry, S. Hews, J. D. Nagy, and Y. Kuang, “The dynamics of a delay model of hepatitis B virus infection with logistic hepatocyte growth," Mathematical Biosciences and Engineering, vol. 6, no. 2, pp. 283-299, 2009.

[12] S. A. Gourley, Y. Kuang, and J. D. Nagy, "Dynamics of a delay differential equation model of hepatitis B virus infection," Journal of Biological Dynamics, vol. 2, no. 2, pp. 140-153, 2008.

[13] S. Hews, S. Eikenberry, J. D. Nagy, and Y. Kuang, "Rich dynamics of a hepatitis B viral infection model with logistic hepatocyte growth," Journal of Mathematical Biology, vol. 60, no. 4, pp. 573-590, 2010.

[14] M. A. Nowak, S. Bonhoeffer, A. M. Hill, R. Boehme, H. C. Thomas, and H. Mcdade, "Viral dynamics in hepatitis B virus infection," Proceedings of the National Acadamy of Sciences of the United States of America, vol. 93, no. 9, pp. 4398-4402, 1996.

[15] J. Pang and J.-A. Cui, "Analysis of a hepatitis B viral infection model with immune response delay," International Journal of Biomathematics, vol. 10, no. 2, Article ID 1750020, 2017.

[16] A. Tridane, K. Hattaf, R. Yafia, and F. A. Rihan, "Mathematical modeling of hbv with the antiviral therapy for the immunocompromised patients," Communications in Mathematical Biology and Neuroscience, vol. 2016, 31 pages, 2016.

[17] Y. Wang and X. Liu, "Dynamical behaviors of a delayed HBV infection model with logistic hepatocyte growth, cure rate and CTL immune response," Japan Journal of Industrial and Applied Mathematics, vol. 32, no. 3, pp. 575-593, 2015.

[18] N. Yousfi, K. Hattaf, and A. Tridane, "Modeling the adaptive immune response in HBV infection," Journal of Mathematical Biology, vol. 63, no. 5, pp. 933-957, 2011.

[19] A. Meskaf, K. Allali, and Y. Tabit, "Optimal control of a delayed hepatitis B viral infection model with cytotoxic T-lymphocyte and antibody responses," International Journal of Dynamics and Control, vol. 5, no. 3, pp. 893-902, 2017.

[20] J. E. Forde, S. M. Ciupe, A. Cintron-Arias, and S. Lenhart, "Optimal control of drug therapy in a hepatitis B model," Applied Sciences (Switzerland), vol. 6, no. 8, article no. 219, 2016.

[21] A. M. Elaiw, M. A. Alghamdi, and S. Aly, "Hepatitis B virus dynamics: Modeling, analysis, and optimal treatment scheduling," Discrete Dynamics in Nature and Society, vol. 2013, Article ID 712829, 2013.

[22] P. Tchinda Mouofo, J. J. Tewa, B. Mewoli, and S. Bowong, "Optimal control of a delayed system subject to mixed controlstate constraints with application to a within-host model of hepatitis virus B," Annual Reviews in Control, vol. 37, no. 2, pp. 246-259, 2013.

[23] S. M. Ciupe, R. M. Ribeiro, P. W. Nelson, G. Dusheiko, and A. S. Perelson, "The role of cells refractory to productive infection in acute hepatitis B viral dynamics," Proceedings of the National Acadamy of Sciences of the United States of America, vol. 104, no. 12, pp. 5050-5055, 2007.

[24] G. K. Michalopoulos and M. C. DeFrances, "Liver regeneration," Science, vol. 276, no. 5309, pp. 60-65, 1997.

[25] M. A. Nowak and R. M. May, Virus Dynamics, Oxford University Press, London, UK, 2000.

[26] S. A. Whalley, J. M. Murray, D. Brown et al., "Kinetics of acute hepatitis B virus infection in humans," The Journal of Experimental Medicine, vol. 193, no. 7, pp. 847-853, 2001.

[27] M.-P. Bralet, S. Branchereau, C. Brechot, and N. Ferry, "Cell lineage study in the liver using retroviral mediated gene transfer: Evidence against the streaming of hepatocytes in normal liver," The American Journal of Pathology, vol. 144, no. 5, pp. 896-905, 1994.

[28] R. A. Macdonald, “"Lifespan” of Liver Cells: Autoradiographic Study Using Tritiated Thymidine in Normal, Cirrhotic, and Partially Hepatectomized Rats," JAMA Internal Medicine, vol. 107, no. 3, pp. 335-343, 1961.

[29] S. M. Ciupe, R. M. Ribeiro, P. W. Nelson, and A. S. Perelson, "Modeling the mechanisms of acute hepatitis B virus infection," Journal of Theoretical Biology, vol. 247, no. 1, pp. 23-35, 2007.

[30] M. A. Nowak and R. M. May, Virus Dynamics: Mathematics Principles of Immunology and Virology, Oxford University Press, London, UK, 2000.

[31] S. M. Ciupe, R. M. Ribeiro, and A. S. Perelson, "Antibody responses during hepatitis B viral infection," PLoS Computational Biology, vol. 10, no. 7, Article ID e1003730, 2014.

[32] R. Ahmed and D. Gray, "Immunological memory and protective immunity: Understanding their relation," Science, vol. 272, no. 5258 , pp. 54-60, 1996.

[33] W. H. Fleming and R. W. Rishel, Deterministic and Stochastic Optimal Control, vol. 1, Springer, New York, NY, USA, 1975.

[34] D. L. Lukes, Differential Equations: Classical to Controlled, Mathematics in Science and Engineering, Academic Press, New York, NY. USA, 1982.

[35] L. Gollmann, D. Kern, and H. Maurer, "Optimal control problems with delays in state and control variables subject to mixed control-state constraints," Optimal Control Applications and Methods, vol. 30, no. 4, pp. 341-365, 2009.

[36] A. B. Gumel, P. N. Shivakumar, and B. M. Sahai, "A mathematical model for the dynamics of HIV-1 during the typical course of infection," Nonlinear Analysis: Theory, Methods \& Applications, vol. 47, no. 3, pp. 1773-1783, 2001.

[37] J. Karrakchou, M. Rachik, and S. Gourari, "Optimal control and infectiology: application to an HIV/AIDS model," Applied Mathematics and Computation, vol. 177, no. 2, pp. 807-818, 2006.

[38] L. Chen, K. Hattaf, and J. Sun, "Optimal control of a delayed SLBS computer virus model," Physica A: Statistical Mechanics and its Applications, vol. 427, pp. 244-250, 2015.

[39] K. Hattaf and N. Yousfi, "Optimal control of a delayed HIV infection model with immune response using an efficient numerical method," ISRN biomathematics, vol. 2012, Article ID 215124, 7 pages, 2012.

[40] H. Laarabi, A. Abta, and K. Hattaf, "Optimal Control of a Delayed SIRS Epidemic Model with Vaccination and Treatment," Acta Biotheoretica, vol. 63, no. 2, pp. 87-97, 2015.

[41] L. Boglione, G. Cariti, G. Di Perri, and A. D’Avolio, "Sequential therapy with entecavir and pegylated interferon in a cohort of young patients affected by chronic hepatitis B," Journal of Medical Virology, vol. 88, no. 11, pp. 1953-1959, 2016. 


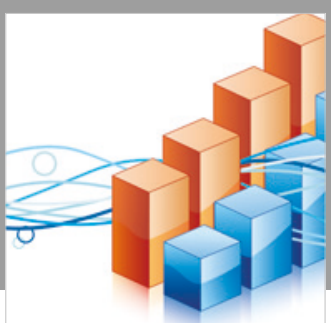

Advances in

Operations Research

\section{-n-m}
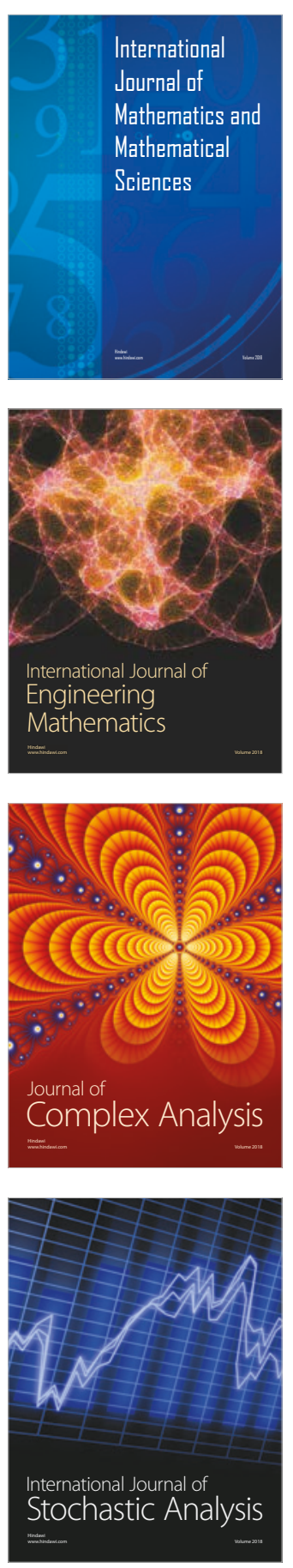
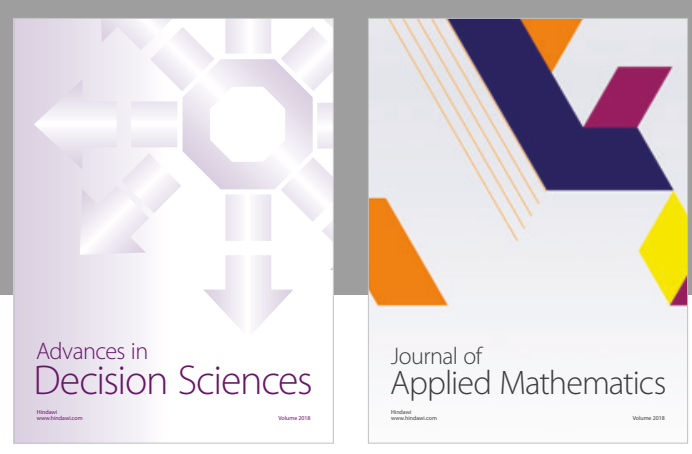

Journal of

Applied Mathematics
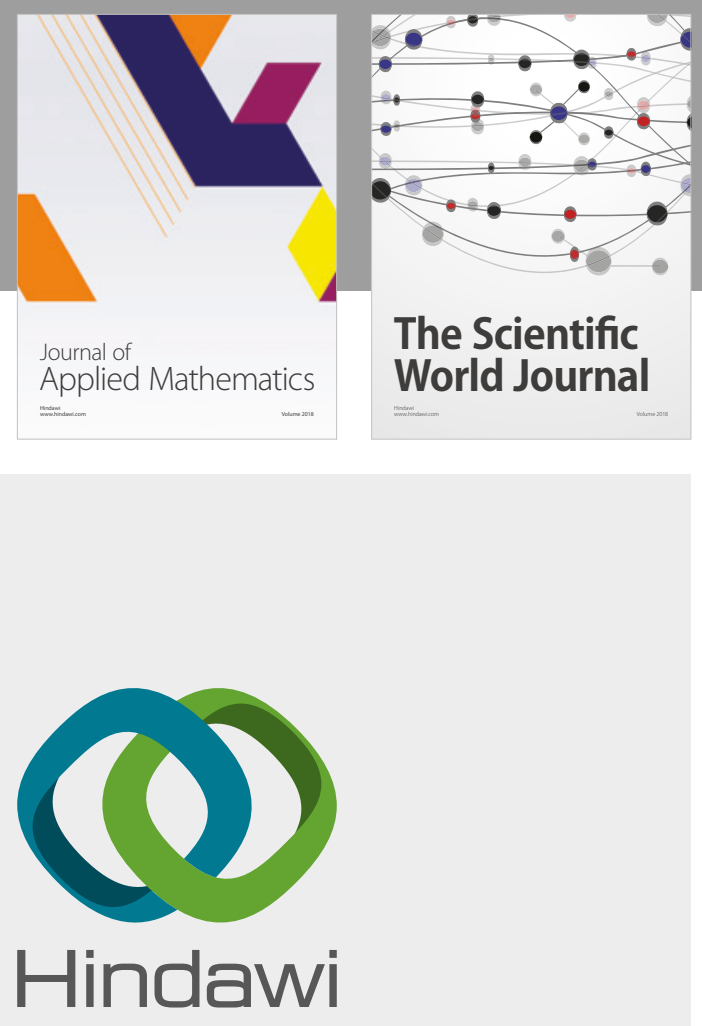

Submit your manuscripts at

www.hindawi.com

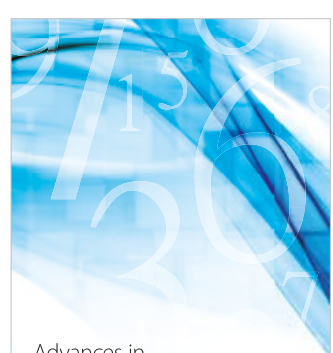

Advances in
Numerical Analysis
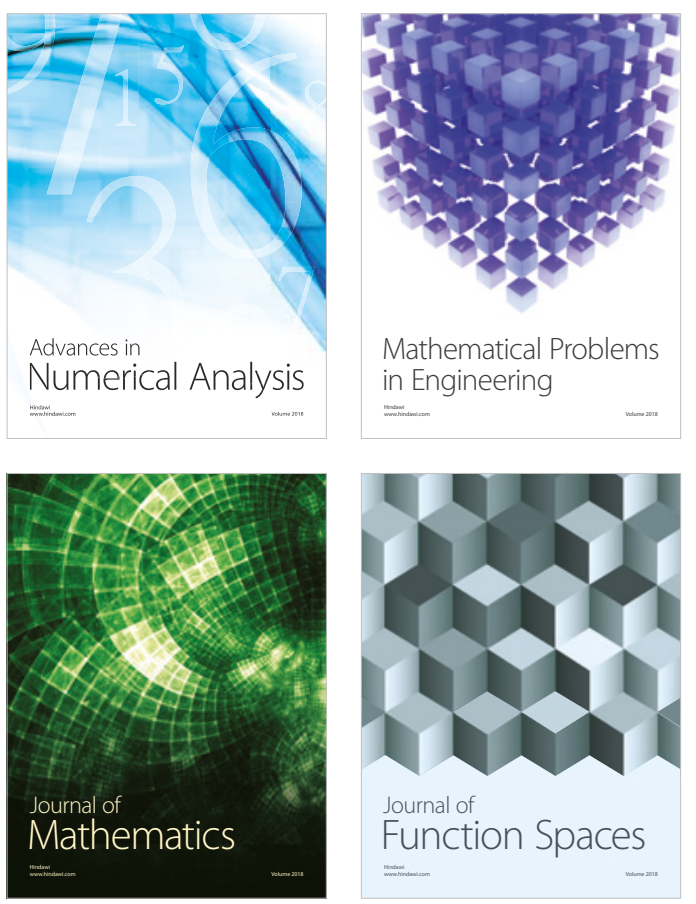

Mathematical Problems in Engineering

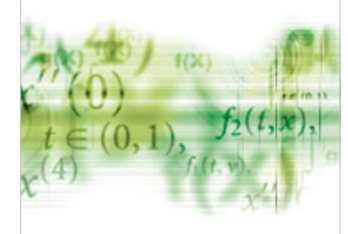

International Journal of

Differential Equations

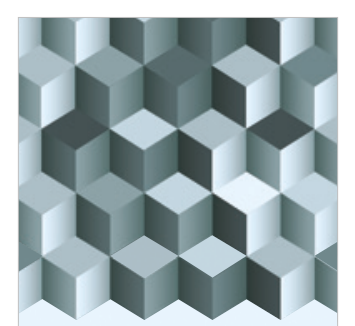

Journal of

Function Spaces

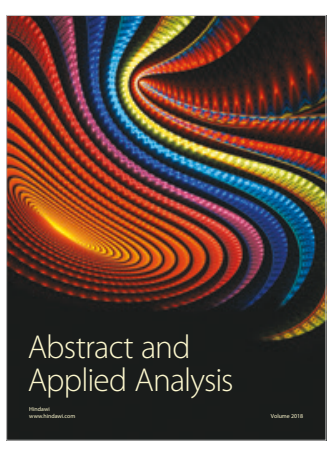

The Scientific

World Journal

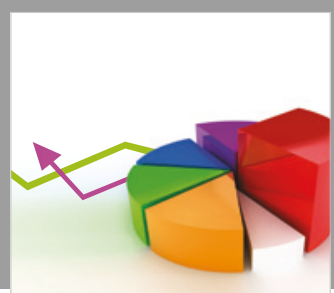

Journal of

Probability and Statistics
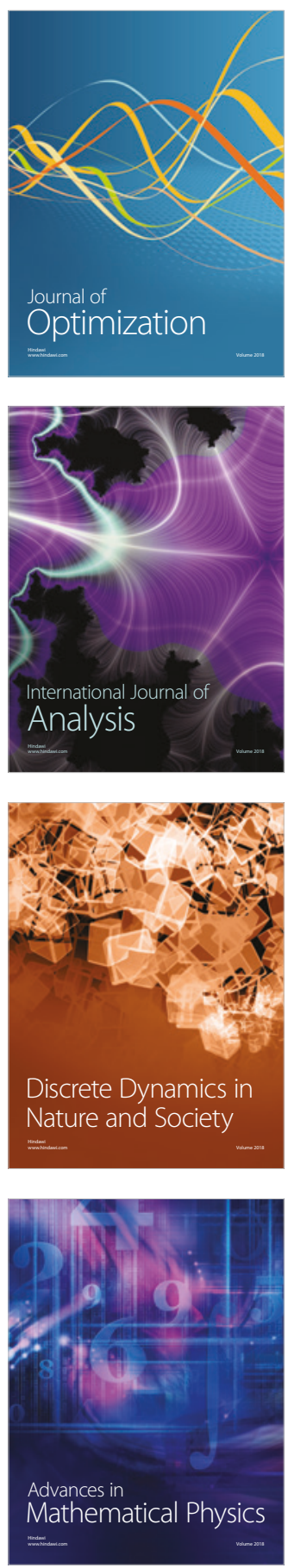